\title{
Factorization and escorting in the game-theoretical approach to non-extensive entropy measures
}

\author{
Flemming Topsøe \\ Department of Mathematics, University of Copenhagen, topsoe@math.ku.dk
}

(Date textdate; Received textdate; Revised textdate; Accepted textdate; Published textdate)

\begin{abstract}
The game-theoretical approach to non-extensive entropy measures of statistical physics is based on an abstract measure of complexity from which the entropy measure is derived in a natural way. A wide class of possible complexity measures is considered and a property of factorization investigated. The property reflects a separation between the system being observed and the observer. Apparently, the property is also related to escorting. It is shown that only those complexity measures which are connected with Tsallis entropy have the factorization property.
\end{abstract}




\section{ENTROPY VIA COMPLEXITY}

The present contribution should be seen in the context of the overall goal: to give operational definitions of entropy and related quantities which cover the classical as well as non-extensive settings, thereby leading to an understanding of which of these quantities, in particular which entropy measures, are relevant for statistical physics.

The findings here reported constitute a companion to the game theoretical approach discussed in Topsøe [3]. For the convenience of the reader, we repeat some of the essentials. The key objects of the approach are the alphabet $A$, taken to be discrete, the strategy set $S_{I}$ of Player I ("Nature"), also referred to as the preparation, a strategy set $S_{I I}$ for Player II ("the physicist") and a real, or extended real valued complexity function $\Phi$ defined on the product set $S_{I} \times S_{I I}:(P, Q) \curvearrowright \Phi(P \| Q)$. The strategy set $S_{I I}$ is here taken to be the set of all probability distributions over $A$ and $S_{I}$ is assumed to be a subset of $S_{I I}$. A typical element in $S_{I}$ is denoted $P$ and a typical element in $S_{I I}$ is denoted $Q$. Point probabilities are denoted by a subscript $i$ which then ranges over $A$, e.g. $P=\left(p_{i}\right)_{i \in A}, Q=\left(q_{i}\right)_{i \in A}$.

The basic axiom we shall work with concerns the complexity function and states that for each $P \in S_{I}$, the minimum over $Q \in S_{I I}$ of $\Phi(P \| Q)$ is finite and achieved for $Q=P$ and for no other probability distribution.

The entropy function $S_{\Phi}$ is defined by

$$
S_{\Phi}(P)=\inf _{Q \in S_{I I}} \Phi(P \| Q)
$$

The essential requirement imposed on the complexity function is then that the bi-implication

$$
\Phi(P \| Q)=S_{\Phi}(P) \Leftrightarrow Q=P
$$

holds for any $P \in S_{I}$. Divergence $D(P \| Q)$ is the difference between complexity and entropy:

$$
D_{\Phi}(P \| Q)=\Phi(P \| Q)-S_{\Phi}(P)=\Phi(P \| Q)-\Phi(P \| P) .
$$

The game alluded to in the title is the two-person zero-sum game for which Player I aims at high complexity and Player II at low complexity. As is evident from (11), a maximum entropy distribution is the same as a maximin strategy, i.e. as an optimal strategy for Player I. We are thus led directly to Jaynes maximum entropy principle (MaxEnt) and the game theoretical approach can to some extent be said to explain that principle. A deeper 
understanding requires that also optimal strategies for Player II are taken into consideration. For details, see [3].

We claim that the game theoretical view offers sound principles which appear more natural and at a deeper level than MaxEnt itself. It has to be pointed out, however, that the approach does not give a clue to which entropy measures appear naturally in physics. Some results which address this problem from the game theoretical point of view are contained in [3] and here we continue research in this direction. We focus on the triple $(\Phi, S, D)$ of complexity, entropy and divergence and limit the study to triples of the form

$$
\begin{aligned}
\Phi(P \| Q) & =\sum_{i \in A}\left(q_{i} f\left(\frac{p_{i}}{q_{i}}\right)-f\left(p_{i}\right)\right), \\
S(P) & =-\sum_{i \in A} f\left(p_{i}\right), \\
D(P \| Q) & =\sum_{i \in A} q_{i} f\left(\frac{p_{i}}{q_{i}}\right),
\end{aligned}
$$

where $f$, the generator, is a real-valued analytic and strictly convex function on $[0, \infty[$ such that $f(0)=f(1)=0$ and such that the normalization condition $f^{\prime}(1)=1$ is fulfilled. We may put $f(\infty)=\infty$. The condition of analyticity, which does not appear in [3], guarantees that $f$ is smooth and that the behavior of $f$ in one interval, say in $[0,1]$, determines the behavior in the entire interval $[0, \infty]$.

It is easy to check, by appeal to Jensen's inequality, that with $S$ and $D$ given by (5) and (6), $S=S_{\Phi}$ and $D=D_{\Phi}$.

Often, it is advantageous to work with the dual generator $\tilde{f}$. This is the function given by

$$
\widetilde{f}(x)=x f\left(\frac{1}{x}\right), 0 \leq x \leq \infty .
$$

The formulas (41)-(6) expressed in terms of the dual generator give the expressions

$$
\begin{aligned}
\Phi(P \| Q) & =\sum_{i \in A} p_{i}\left(\tilde{f}\left(\frac{q_{i}}{p_{i}}\right)-\tilde{f}\left(\frac{1}{p_{i}}\right)\right), \\
S(P) & =-\sum_{i \in A} p_{i} \tilde{f}\left(\frac{1}{p_{i}}\right) \\
D(P \| Q) & =\sum_{i \in A} p_{i} \tilde{f}\left(\frac{q_{i}}{p_{i}}\right) .
\end{aligned}
$$

We realize that a very wide class of potentially interesting entropy measures are of the form here considered. In the classical case, leading to the Boltzmann-Gibbs-Shannon entropy, 
we have $f(x)=x \ln x$ and $\widetilde{f}(x)=\ln \frac{1}{x}$. Further cases work with deformed logarithms. A two-parameter version of these, taken from [3], are given by

$$
\ln _{\alpha, \beta} x=\left\{\begin{array}{l}
\frac{x^{\beta}-x^{\alpha}}{\beta-\alpha} \text { if } \beta \neq \alpha, \\
x^{\alpha} \ln x \text { if } \beta=\alpha .
\end{array}\right.
$$

With appropriate choice of parameters $(-1<\alpha \leq 0,0 \leq \beta$ or the symmetric choice) this leads to the family of generators given by

$$
f_{\alpha, \beta}(x)=x \ln _{\alpha, \beta}(x) \text { with dual } \tilde{f}_{\alpha, \beta}(x)=\ln _{\alpha, \beta} \frac{1}{x} .
$$

With $(\alpha, \beta)=(1-q, 0)$ for a positive value of the parameter $q$, we are led to Tsallis $q$ entropy, cf. Tsallis [4]. Listing also the associated complexity and divergence functions we find that they are given by the expressions:

$$
\begin{aligned}
\Phi_{q}(P \| Q) & =\frac{1}{1-q} \sum_{i \in A} p_{i}^{q}\left(1-q_{i}^{1-q}\right), \\
S_{q}(P) & =\frac{1}{1-q} \sum_{i \in A} p_{i}\left(p_{i}^{q-1}-1\right)=\frac{1}{1-q}\left(\sum_{i \in A} p_{i}^{q}-1\right), \\
D_{q}(P \| Q) & =\frac{1}{1-q} \sum_{i \in A} p_{i}\left(1-\left(\frac{p_{i}}{q_{i}}\right)^{q-1}\right)=\frac{1}{1-q}\left(1-\sum_{i \in} p_{i}\left(\frac{p_{i}}{q_{i}}\right)^{q-1}\right) .
\end{aligned}
$$

A significant feature is the factorization property of (13). Note that this structural property is not immediately visible just looking at the entropy function (14).

\section{FACTORIZATION}

Let us have a closer look at the factorization property and investigate for which generators $f$ the associated complexity function factorizes. In more detail, with $\Phi$ of the form (4), what we search for are functions $\xi$ and $\zeta$ such that

$$
\Phi(P \| Q)=\sum_{i \in A} \xi\left(p_{i}\right) \zeta\left(q_{i}\right)
$$

holds generally. We call $\xi$ the escort function. It acts as a kind of mean-value generator and invites for escorting, which is the consideration, along with any distribution $P=\left(p_{i}\right)_{i \in A}$ of the associated escort distribution with point probabilities $\left(\xi\left(p_{i}\right) / Z\right)_{i \in A}$ where $Z$ is the appropriate constant of normalization. The consideration of escort probabilities occurs in 
connection with MaxEnt but presents in itself problems regarding the proper physical interpretation. The literature can be traced from the recent contribution by Ferri, Martínez and Plastino, cf. 11] and, for critical comments, from Shalizi [2]. The function $\zeta$ is the surprise factor. This may also be thought of as self-information. We require that $\xi(1)=1$ and $\zeta(1)=0$ and that both functions are analytic in $[0, \infty[$. Thus, we demand that escorting does not change a deterministic distribution and that the surprise value associated with the occurrence of a deterministic event is 0 . The escort function and the surprise function need in fact only be given for arguments in $[0,1]$. However, the requirement of analyticity is a requirement that these functions have natural extensions to functions defined for any positive argument. Therefore, we may as well assume that the functions are defined from the outset on the whole interval $[0, \infty[$.

Now assume that $\Phi$ given by (4) factorizes as described above. Then, from the formula

$$
\begin{aligned}
D(P \| Q) & =\Phi(P \| Q)-\Phi(P \| P)=\sum_{i \in A} \xi\left(p_{i}\right)\left(\zeta\left(q_{i}\right)-\zeta\left(p_{i}\right)\right) \\
& =\sum_{i \in A} p_{i} \tilde{f}\left(\frac{q_{i}}{p_{i}}\right)
\end{aligned}
$$

we realize that

$$
\xi(x)((\zeta(y)-\zeta(x)))=x \widetilde{f}\left(\frac{y}{x}\right) .
$$

In fact, by simple considerations, also using a continuity argument, this is first established for $x$ and $y$ in the unit interval. The general validity then follows by analyticity considerations.

Putting first $x=1$, then $y=1$ in (17) we realize that $\xi$ and $\zeta$ can be identified in terms of $\tilde{f}$. In fact,

$$
\zeta=\widetilde{f} \text { and } \xi(x)=\frac{x \widetilde{f}\left(\frac{1}{x}\right)}{-\widetilde{f}(x)}
$$

Inserted into (17) we see that

$$
\frac{x \widetilde{f}\left(\frac{1}{x}\right)}{-\widetilde{f}(x)}(\widetilde{f}(y)-\widetilde{f}(x))=x \widetilde{f}\left(\frac{y}{x}\right),
$$

hence

$$
\widetilde{f}\left(\frac{1}{x}\right)(\widetilde{f}(x)-\widetilde{f}(y))=\widetilde{f}(x) \widetilde{f}\left(\frac{y}{x}\right)
$$

or

$$
\widetilde{f}\left(\frac{1}{x}\right)(\widetilde{f}(x)-\widetilde{f}(x y))=\tilde{f}(x) \tilde{f}(y)
$$


Then

$$
\begin{aligned}
\frac{\tilde{f}(x)+\widetilde{f}(y)-\widetilde{f}(x y)}{\widetilde{f}(x) \widetilde{f}(y)} & =\frac{1}{\widetilde{f}(x)}+\frac{\widetilde{f}(x)-\widetilde{f}(x y)}{\widetilde{f}(x) \widetilde{f}(y)} \\
& =\frac{1}{\widetilde{f}\left(\frac{1}{x}\right)}+\frac{1}{\widetilde{f}(x)} \\
& =\alpha,
\end{aligned}
$$

a constant (because of the dependence on $x$ only in the last expression and the symmetry in $x$ and $y$ of the expression we started out with). In case $\alpha=0$ we are soon led to conclude that then $\tilde{f}(x)=\ln \frac{1}{x}$ and we are faced with the classical Boltzmann-Gibbs-Shannon case. When $\alpha \neq 0$, we use the functional equation

$$
(1-\alpha \widetilde{f}(x y))=(1-\alpha \widetilde{f}(x))(1-\alpha \widetilde{f}(y))
$$

and conclude, as $\tilde{f}^{\prime}(1)=-1$, that $1-\alpha \tilde{f}(x)=x^{\alpha}$, hence

$$
\widetilde{f}(x)=\frac{1-x^{\alpha}}{\alpha}=\ln _{-\alpha, 0} \frac{1}{x} .
$$

As $f$, hence also $\tilde{f}$, is required to be convex, we must conclude that $\alpha<1$. We realize that we are faced with the Tsallis family (with parameter $q=1-\alpha$ ).

To sum up, we have proved:

Theorem 1 A complexity function of the form (4) factorizes if and only if it is related to one of the Tsallis entropies, cf. (13)-(14).

\section{CONCLUSIONS}

Factorization of the complexity function appears important as it separates the two sides of the game, that of nature and that of the physicist. The property can be used to characterize the family of entropy measures suggested by Tsallis.

Though our results are, hopefully, indicative of central issues for fundamentals of nonextensive statistical physics, operational interpretations are not yet in place. The game

theoretical approach may well be helpful in this connection, see [3] for some further results, 
especially on equilibrium, and for open problems to look into.

[1] G L Ferri, S Martínez, and A Plastino. Equivalence of the four versions of Tsallis's statistics. Journal of Statistical Mechanics: Theory and Experiment, 2005(04):P04009, 2005.

[2] C. R. Shalizi. Tsallis Statistics, Statistical Mechanics for Non-extensive Systems and LongRange Interactions. Available at http://cscs.umich.edu/ crshalizi/notebooks/tsallis.html.

[3] F. Topsøe. Entropy and Equilibrium via Games of Complexity. Physica A, 340/1-3:11-31, 2004.

[4] C. Tsallis. Possible generalization of Boltzmann-Gibbs statistics. J. Stat. Physics, 52:479, 1988. See http://tsallis.cat.cbpf.br/biblio.htm for an extensive and updated bibliography. 